\title{
Compuestos antioxidantes y su bioaccesibilidad in vitro de la zanahoria (Daucus carota): cambios por procesos térmicos
}

\section{Antioxidant compounds and its in vitro bioaccessibility of carrot (Daucus carota): changes by thermal processes}

\author{
Nohemí Espinoza-Gayosso ${ }^{a}$, Esther Ramírez-Moreno ${ }^{b}$, Nelly del Socorro Cruz-Cansino ${ }^{c}$, \\ Alicia Cervantes-Elizarrarás ${ }^{d}$, Quinatzin Y. Zafra-Rojas ${ }^{e}$
}

\begin{abstract}
:
The carrot (Daucus carota) belongs to the Umbelliferae family, is vegetable rich in $\beta$-carotenes and other antioxidants. The aim of the review was to analyze the effect of thermal processing of carrot on antioxidant compounds and their bioaccessibility. The thermal treatments vary according to the temperature and time conditions to which the food is subjected. Researchers have reported the application of thermal treatments to carrots such as boiling, pasteurization, ultra-pasteurization, commercial sterilization, blanching, pressure cooking and frying. There are significant changes in the colour parameters when the carrots are blanched, the values of $a^{*}$ and $b^{*}$ decreased with respect to the untreated samples. The content of phenolic compounds and their bioaccessibility decreased with the blanching and pasteurization. Egmont Gold carrot treated at $80^{\circ} \mathrm{C}$ increased the concentration of ascorbic acid $(318.82 \pm 18.40$ $\mu \mathrm{g} / \mathrm{g} \mathrm{dw}$ (dry weight)) by $23.84 \%$, with respect to the sample at room temperature $(258.12 \pm 32.56 \mu \mathrm{g} / \mathrm{g}$ dw), due to the inactivation of the enzyme ascorbic acid oxidase. Pressure cooking $(5134.5 \pm 75.3 \mu \mathrm{g} / 100 \mathrm{~g}$ fw (fresh weight)), boiled $(6740.3 \pm 58.6 \mu \mathrm{g} / 100 \mathrm{~g}$ $\mathrm{fw})$, frying $(5926.4 \pm 106.6 \mu \mathrm{g} / 100 \mathrm{~g} \mathrm{fw})$, blanched $(87.70 \pm 2.75 \mu \mathrm{g} / \mathrm{g} \mathrm{fw})$ and drying $(882-1079 \mathrm{mg} / \mathrm{kg}$ dry mass) of the carrot, caused a decrease in carotenoids (2.53-31.58\%). On the contrary, when was analyzed the in vitro bioaccessibility, pressure cooking, boiling and frying increased the bioaccessibility of carotenoids $(29.80 \%, 25.45 \%$ and $38.47 \%$, respectively) compared to carrots without treatment $(16.80 \%)$, this behaviour also occurs in pasteurized, sterilized, ultra-pasteurized and dehydrated carrot pulp. However, the bioaccessibility of carrot antioxidant compounds depends on the polysaccharides of the cell membrane, proteins and other factors of the food matrix.
\end{abstract}

Keywords:

carrot, juice, carotenoids, colour, thermal treatments

\section{Resumen:}

La zanahoria (Daucus carota) pertenece a la familia de las Umbelíferas, es un vegetal rico en $\beta$-carotenos y otros antioxidantes. El objetivo de la revisión fue analizar el efecto del procesamiento térmico de la zanahoria sobre los compuestos antioxidantes y su bioaccesibilidad. Los tratamientos térmicos varían de acuerdo a las condiciones de temperatura y tiempo a los que son sometidos los alimentos. Investigaciones han reportado la aplicación de tratamientos térmicos en zanahoria como hervido, pasteurización, ultrapasteurización, esterilización comercial, escaldado, cocción a presión y freído. Existen cambios significativos en los parámetros de color cuando las zanahorias son escaldadas, los valores de $a^{*}$ y $b^{*}$ disminuyeron respecto a las muestras no tratadas. El contenido de compuestos fenólicos y su bioaccesibilidad se reduce con el escaldado y la pasteurización. La zanahoria Egmont Gold tratada a $80^{\circ} \mathrm{C}$ incrementó la concentración de ácido ascórbico $(318.82 \pm 18.40 \mu \mathrm{g} / \mathrm{g}$ ps (peso seco)) en $23.84 \%$, respecto a la muestra a temperatura ambiente $(258.12 \pm 32.56 \mu \mathrm{g} / \mathrm{g} \mathrm{ps})$, debido a la inactivación de la enzima ácido ascórbico oxidasa. La cocción a presión $(5134.5 \pm 75.3 \mu \mathrm{g} / 100 \mathrm{~g} \mathrm{pf}$ (peso fresco)), hervido $(6740.3 \pm 58.6 \mu \mathrm{g} / 100 \mathrm{~g} \mathrm{pf})$, freído $(5926.4 \pm 106.6 \mu \mathrm{g} / 100 \mathrm{~g}$ pf), escaldado

\footnotetext{
a Universidad Autónoma del Estado de Hidalgo, México, https://orcid.org/0000-0002-3504-9432, Email: es340967@uaeh.edu.mx

b Universidad Autónoma del Estado de Hidalgo, México, https://orcid.org/0000-0002-9928-8600, Email: esther_ramirez@uaeh.edu.mx

c Universidad Autónoma del Estado de Hidalgo, México, https://orcid.org/0000-0002-6771-3684, Email: ncruz@uaeh.edu.mx

d Universidad Autónoma del Estado de Hidalgo, México, https://orcid.org/0000-0002-1432-2882, Email: alicia_cervantes@uaeh.edu.mx

Autor de Correspondencia, Universidad Autónoma del Estado de Hidalgo, México, https://orcid.org/0000-0002-5295-9972, Email:
} quinatzin_zafra@uaeh.edu.mx 
$(87.70 \pm 2.75 \mu \mathrm{g} / \mathrm{g}$ pf) y secado $(882-1079 \mathrm{mg} / \mathrm{kg}$ masa seca) de la zanahoria, provocó una disminución de carotenoides $(2.53-31.58 \%)$. Por el contrario, cuando se analizó la bioaccesibilidad in vitro, la cocción a presión, hervido y freído incrementaron la bioaccesibilidad de los carotenoides $(29.80 \%, 25.45 \%$ y $38.47 \%$, respectivamente) en comparación con la zanahoria sin tratamiento (16.80\%), este comportamiento también ocurre en la pulpa de zanahoria pasteurizada, esterilizada, ultrapasteurizada y deshidratada. Sin embargo, la bioaccesibilidad de los compuestos antioxidantes de la zanahoria depende de los polisacáridos de la membrana celular, proteínas y otros factores de la matriz alimentaria.

\section{Palabras Clave:}

Zanahoria, jugo, carotenoides, color, tratamientos térmicos

\section{Introducción}

La zanahoria (Daucus carota) es una hortaliza y pertenece a la familia de las umbelíferas, es originaria de Asia y cultivada en todo el mundo. De acuerdo a su coloración, la zanahoria se puede encontrar en tonos amarillos, rojos, morados, blancos y anaranjados. ${ }^{1}$ En fresco, la zanahoria es reconocida por su alto contenido en carotenoides como $\alpha$-carotenos (2744.8 \pm 207.4 $\mu \mathrm{g} / 100 \mathrm{~g}$ peso fresco) y $\beta$-carotenos (4982.0 \pm 362.6 $\mu \mathrm{g} / 100 \mathrm{~g}$ peso fresco). ${ }^{2}$ Además contiene vitaminas como vitamina A (Eq. Retinol) $(1.346 \mu \mathrm{g} / 100 \mathrm{~g})$, vitamina C (6 $\mathrm{mg} / 100 \mathrm{~g})$ y vitamina $\mathrm{B} 3$ (niacina) $(0.6 \mathrm{mg} / 100 \mathrm{~g})$ y algunos minerales, entre los cuales destacan el potasio $(255 \mathrm{mg} / 100 \mathrm{~g})$, sodio $(77 \mathrm{mg} / 100 \mathrm{~g})$ y calcio (41 $\mathrm{mg} / 100 \mathrm{~g})^{3}$, también contiene polifenoles $(1.75 \mathrm{mg}$ $\mathrm{EAG} / 100 \mathrm{~g}$ ps (equivalentes de ácido gálico/100 g en peso seco $)^{4}$, flavonoides $(35.9 \pm 0.97 \mathrm{mg} \mathrm{CAT} / 100 \mathrm{~g}$ ps (equivalentes de catequina/100 g peso seco en cáscara de zanahoria) y antocianinas (1653.8 $\pm 183.1 \mathrm{mg} \mathrm{C} 3 \mathrm{G}$ (cianidina-3-O-glucósido)/100 g ps en zanahoria morada), los cuales contribuyen a una elevada capacidad antioxidante medida por DPPH $(7198.7 \pm 1027.3 \mathrm{mg}$ de equivalentes de Trolox/100 g peso seco) y FRAP (9257.2 $\pm 1537.0 \mathrm{mg}$ de equivalentes de Trolox/100 g peso seco) en zanahoria morada. ${ }^{5,6}$ El consumo de alimentos ricos en compuestos bioactivos está asociado con la prevención de enfermedades cardiovasculares, óseas, dérmicas y cáncer (próstata, mama, cuello uterino, ovario y colorrectal). ${ }^{7,8}$ La zanahoria presenta alta cantidad de vitamina A y ayuda a prevenir problemas visuales como cataratas, sequedad ocular, conjuntivitis y ceguera nocturna. ${ }^{9}$

La zanahoria puede consumirse en fresco o cocida, en infusión, para preparar sopas, ensaladas y pasteles, sin embargo también es consumida como jugo, asada, frita 0 en puré. ${ }^{10}$ Estos tratamientos térmicos empleados en los alimentos inactivan microorganismos patógenos $\mathrm{y} / \mathrm{o}$ enzimas para prolongar la vida útil del producto, no obstante, las elevadas temperaturas pueden desencadenar alteraciones en las características físicas como el color, así como degradación de los compuestos antioxidantes. ${ }^{11,12}$ Por otro lado, se ha reportado que el tratamiento térmico incrementa el contenido de carotenoides (entre 0.53 y $0.83 \mathrm{mg} / \mathrm{kg}$ peso seco) en zanahorias hervidas. ${ }^{4}$ Otros tratamientos térmicos aplicados en la zanahoria han sido la pasteurización, ultrapasteurización, esterilización comercial, escaldado, cocción a presión y freído. ${ }^{1}$

Tomando en consideración la temperatura $\left(63-200^{\circ} \mathrm{C}\right)$ de los tratamientos térmicos antes mencionados, puede mejorar la absorción intestinal (medida como bioaccesibilidad) de los compuestos antioxidantes, debido a la ruptura de las estructuras celulares y las membranas de los organelos, lo cual permite la liberación de compuestos teniendo efectos positivos a la salud, como disminuir el estrés oxidativo, y contribuye a reducir el padecer enfermedades gástricas, respiratorias, neurológicas y del sistema endocrino. ${ }^{14,15}$

Según Saura-Calixto et al. ${ }^{16}$ la bioaccesibilidad se define como la cantidad de un constituyente de alimento que está presente en el intestino, como consecuencia de la liberación de este componente de la matriz del alimento sólido, y puede ser capaz de pasar a través de la barrera intestinal. Solamente los polifenoles liberados de la matriz del alimento por la acción de enzimas digestivas (intestino delgado) y la microbiota (intestino grueso) son bioaccesibles en el intestino y por lo tanto potencialmente biodisponibles. Por lo anterior, el objetivo de la revisión fue analizar el efecto del procesamiento térmico de la zanahoria (Daucus carota) sobre los compuestos antioxidantes y su bioaccesibilidad.

\section{Características de la zanahoria}

La zanahoria (Daucus carota) es una hortaliza de raíz larga de color naranja, sin embargo, existen zanahorias con coloraciones blancas, rojas, moradas y amarillas. ${ }^{1} \mathrm{La}$ zanahoria blanca, conocida también como arracacha, apio criollo, racacha, virraca o mandioquiña salsa, es una planta originaria de los Andes $^{17}$, mientras que la zanahoria roja es nativa de la India y China, suelen denominarse zanahorias "pakistaníes", la característica principal de esta hortaliza es su cáscara roja y la pulpa naranja. ${ }^{18}$ La zanahoria morada inicialmente se cultivó en Afganistán, su exterior es de tonalidad púrpura intensa, el centro de la raíz es amarillo o naranja y existe evidencia de que esta zanahoria fue la original, las variedades de 
color naranja se originaron tiempo después, cuando el cultivo llegó a otras regiones. ${ }^{19}$

Por otro lado, el sabor de la zanahoria es insípido y se debe a que lo constituyen varios componentes fenólicos, ácidos clorogénicos, junto a varios terpenos y se caracteriza por poseer una textura leñosa. ${ }^{20,21}$ Las hojas tienen los peciolos largos, doble o triplemente pinnadopartidas, y toman una disposición en roseta, mientras que las semillas son pequeñas, en diaquenio, provistas de unos aguijones curvados en el extremo. ${ }^{22}$ Las flores son de color blanco, pequeñas y se encuentran agrupados en tallos en forma de sombrilla, su raíz es fusiforme (Figura 1 ), jugosa y comestible, mide aproximadamente entre 15 y $18 \mathrm{~cm}$ de longitud. ${ }^{7}$

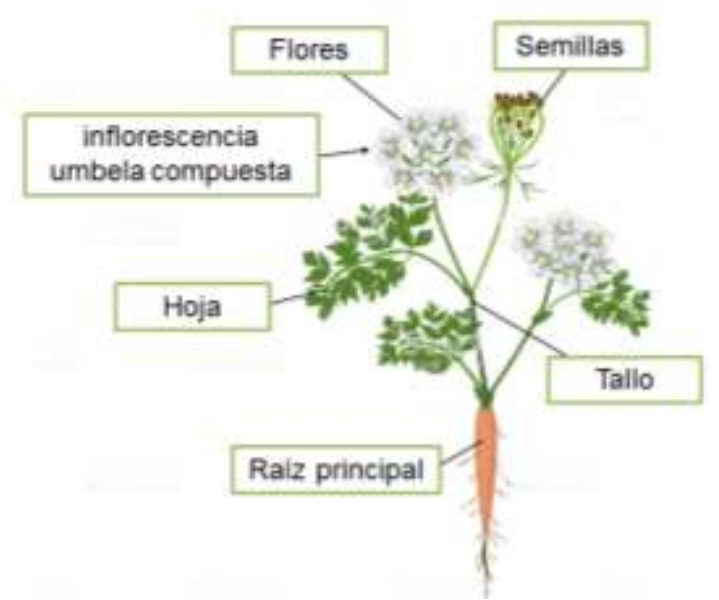

Figura 1. Morfología de la planta de zanahoria ${ }^{23}$

En el 2017, el Ministerio de la Agroindustria ${ }^{24}$ reportó que se producen 16 millones de toneladas de zanahoria en el mundo, entre los cuales China abarca $45 \%$ del total mundial, le siguen la Federación Rusa y Estados Unidos con $4.9 \%$ y $3.7 \%$, respectivamente. En México, la producción de zanahoria en el 2017 fue de 334,013 toneladas $^{9}$ y su consumo es de $7.2 \mathrm{~kg}$ por habitante al año ${ }^{25}$, siendo elevado en comparación con la calabacita, ya que el consumo per cápita anual de ésta es de $2 \mathrm{~kg} .{ }^{26}$ Por lo que incluir la zanahoria en la dieta, se relaciona con efectos positivos a la salud, debido a su contenido nutrimental.

\section{Contenido nutrimental}

La zanahoria contiene en mayor proporción carbohidratos, seguido de fibra, proteínas, minerales y vitaminas (Tabla 1).

\begin{tabular}{|l|l|}
\hline $\begin{array}{l}\text { Tabla 1. Composición nutrimental de la zanahoria } \\
\text { (por cada } 100 \mathrm{~g} \text { peso fresco) }\end{array}$ \\
\hline Elementos & Cantidad \\
\hline Agua (\%) & 88.0 \\
\hline Calorías (kcal) & 41.0 \\
\hline
\end{tabular}

\begin{tabular}{|l|l|}
\hline Proteínas (g) & 1.0 \\
\hline Carbohidratos (g) & 9.6 \\
\hline Lípidos (g) & 0.2 \\
\hline Fibra (g) & 2.8 \\
\hline Cenizas (g) & 1.0 \\
\hline Minerales & 33.0 \\
\hline Calcio (mg) & 35.0 \\
\hline Fósforo (mg) & 0.3 \\
\hline Hierro(mg) & 320.0 \\
\hline Potasio (mg) & 69.0 \\
\hline Sodio (mg) & 20040.0 \\
\hline Vitaminas & 0.07 \\
\hline Vitamina A (U.I) & 0.06 \\
\hline Tiamina (B1) (mg) & 0.98 \\
\hline Riboflavina (B2) (mg) & 5.9 \\
\hline Niacina (B3) (mg) & 0.66 \\
\hline Ácido ascórbico (mg) & \\
\hline Vitamina E (mg) & \\
\hline Modificado de: USDA & \\
\hline
\end{tabular}

Se ha reportado que el consumo de la fibra dietética tiene efectos fisiológicos como el retraso en el vaciamiento gástrico, produce saciedad, disminución en la absorción de colesterol y glucosa. ${ }^{28}$ Respecto a los minerales, el potasio garantiza una adecuada transmisión nerviosa, contracción muscular y cardiaca, tonicidad intracelular, secreción de aldosterona, también interviene en la función renal, metabolismo de hidratos de carbono y síntesis proteica. ${ }^{29}$ Por otro lado, uno de los compuestos representativos de la zanahoria es el caroteno, el cual se transforma en el hígado en vitamina A para producir rodopsina. ${ }^{5}$ Ésta pertenece a los GPCR (receptores acoplados a la proteína $\mathrm{G}$ ) y es la proteína fotorreceptora en la visión, se encuentra en las membranas de los discos de los bastoncillos retinales, su función es la de detectar los fotones que llegan a la retina, los cuales responden al estímulo físico de la luz. ${ }^{30}$

\section{Aplicación de tratamientos térmicos}

Los tratamientos térmicos son métodos físicos que consisten en someter al producto a una fuente de calor por cierto tiempo, antes o después de ser envasado en recipientes de cierre hermético con el fin de lograr una estabilidad biológica. ${ }^{31}$ Por lo tanto, la aplicación de los tratamientos térmicos cambia de acuerdo a las condiciones de temperatura, tiempo y tipo de alimentos. Es amplia la variedad de alimentos en los que puede ser aplicado, sin embargo, en el presente artículo de revisión, solo se mencionan las alteraciones que sufre la zanahoria. 
Uno de los propósitos de estos tratamientos es la inactivación de microorganismos patógenos, así como de enzimas que pueden afectar al alimento, sin embargo, la aplicación de calor puede alterar el color y provoca la degradación de compuestos antioxidantes. ${ }^{11}$ En la Tabla 2 se muestran los tratamientos térmicos como el hervido, pasteurización, ultrapasteurización, esterilización comercial, escaldado, cocción a presión y freído, los cuales han sido aplicados en la zanahoria.

Tabla 2. Condiciones de tratamientos térmicos aplicados en zanahoria

\begin{tabular}{|c|c|c|c|}
\hline $\begin{array}{c}\text { Tratamient } \\
\text { o } \\
\text { térmico }\end{array}$ & Condiciones & Propósito & Cita \\
\hline Hervido & $\begin{array}{l}100^{\circ} \mathrm{C} / 8-23 \\
\min \end{array}$ & $\begin{array}{l}\text { Escasa pérdida de } \\
\text { propiedades } \\
\text { nutritivas }\end{array}$ & $\begin{array}{l}32 \\
33\end{array}$ \\
\hline $\begin{array}{l}\text { Pasteuriza } \\
\text { ción }\end{array}$ & $\begin{array}{l}63^{\circ} \mathrm{C} / 30 \mathrm{~min} \\
72^{\circ} \mathrm{C} / 15 \mathrm{~s}\end{array}$ & $\begin{array}{l}\text { Destrucción de } \\
\text { microorganismos } \\
\text { patógenos viables y } \\
\text { la inactivación de } \\
\text { enzimas }\end{array}$ & $\begin{array}{l}31 \\
34\end{array}$ \\
\hline $\mathrm{UHT}^{*}$ & $\begin{array}{l}135^{-} \\
149^{\circ} \mathrm{C} / 2-8 \mathrm{~s}\end{array}$ & $\begin{array}{l}\text { Destrucción de todos } \\
\text { los microorganismos } \\
\text { viables, } \\
\text { garantizando la } \\
\text { esterilidad comercial } \\
\text { y que sea envasado } \\
\text { asépticamente }\end{array}$ & 34 \\
\hline $\begin{array}{l}\text { Esterilizaci } \\
\text { ón } \\
\text { comercial }\end{array}$ & $\begin{array}{l}120^{\circ} \mathrm{C} / 20 \\
\min \end{array}$ & $\begin{array}{l}\text { Destrucción de todos } \\
\text { los microorganismos } \\
\text { viables de } \\
\text { importancia en la } \\
\text { salud pública y } \\
\text { aquellos capaces de } \\
\text { reproducirse en el } \\
\text { alimento bajo } \\
\text { condiciones de } \\
\text { almacenamiento y } \\
\text { distribución, sin la } \\
\text { condición } \\
\text { refrigeración de }\end{array}$ & 35 \\
\hline Escaldado & $\begin{array}{ll}99 & { }^{\circ} \mathrm{C} / 2-8 \\
\min & \end{array}$ & $\begin{array}{lr}\text { Reducir la } & \text { carga } \\
\text { microbiana } & \text { inicial } \\
\text { mediante } & \text { la } \\
\text { inactivación } & \text { de }\end{array}$ & 4,36 \\
\hline
\end{tabular}

\begin{tabular}{|l|l|l|l|} 
& & $\begin{array}{l}\text { microorganismos } \\
\text { sensibles al calor. }\end{array}$ & \\
\hline $\begin{array}{l}\text { Cocción a } \\
\text { presión }\end{array}$ & $\begin{array}{l}100^{\circ} \mathrm{C} / 15 \\
\text { p.s.i./10 min }\end{array}$ & $\begin{array}{l}\text { Los alimentos } \\
\text { conservan mejor sus } \\
\text { propiedades } \\
\text { nutricionales }\end{array}$ & 37 \\
\hline Freído & $150-200^{\circ} \mathrm{C}$ & -- & 38 \\
\hline
\end{tabular}

*UHT: ultrapasteurización, por sus siglas en inglés (Ultra High Temperature).

Los tratamientos térmicos pueden ir desde 63 hasta los $200^{\circ} \mathrm{C}$ y tiempos que van de los 2 segundos a los 20 minutos. Cabe mencionar que la aplicación de temperatura puede generar cambios estructurales del alimento y en el contenido de antioxidantes.

\section{Cambios por procesos térmicos}

Las investigaciones realizadas sobre la zanahoria (completa, jugo y pulpa) y los tratamientos térmicos, mencionan los cambios en parámetros de color, el incremento o disminución de los compuestos fenólicos, ácido ascórbico, carotenos y su bioaccesibilidad.

\section{Color}

El color en los alimentos es de suma importancia, ya que es el primer atributo que el consumidor toma en cuenta al elegir un alimento. ${ }^{35}$ En un estudio realizado por Wang et al. ${ }^{8}$ se analizó el cambio de color en la zanahoria cuando se aplicó el tratamiento térmico de escaldado $\left(99^{\circ} \mathrm{C}\right)$ a diferentes tiempos (2, 4, 6 y 8 min). Los valores de $L^{*}, a^{*}$ y $b^{*}$ de las zanahorias sin tratamiento (crudas), fueron de $50.97 \pm 0.80,29.67 \pm 2.25$ y $40.14 \pm 2.59$, respectivamente. Por otro lado, los valores de $L^{*}$ de las muestras con tratamiento térmico fueron entre $49.24 \pm 0.51 \mathrm{y}$ $51.96 \pm 1.05$, el parámetro $a^{*}$ tuvo valores de $16.86 \pm 2.66$ a $22.58 \pm 2.39$ y el parámetro $b^{*}$ de $34.32 \pm 1.85$ a $35.82 \pm 1.03$. Se observó que en la luminosidad $\left(L^{*}\right)$ no hubo diferencias significativas entre la muestra sin tratamiento y las muestras escaldadas, mientras que los valores de $a^{*}$ y $b^{*}$ disminuyeron en la zanahoria con tratamiento térmico (se tornaron más rojas) en comparación con las muestras no tratadas. Los carotenoides contenidos en la zanahoria son los responsables de los tonos rojos y amarillos, estos compuestos se encuentran en los cromoplastos, rodeados por una membrana y pared celular, al someter las zanahorias al escaldado, éste destruye la estructura de las células, con lo cual los cromoplastos cristalinos 
entran en el espacio intercelular generando que la zanahoria presente tonalidades más rojizas. ${ }^{39}$

Kaur et al. ${ }^{40}$ analizaron el cambio de color en zanahoria roja y naranja al someterlas a hervido. Los valores de las zanahorias sin tratamiento, es decir, las zanahorias crudas presentaron los siguientes valores para $L^{*}$ (33.81 \pm 0.35 y $34.98 \pm 0.30$, respectivamente), $a^{*}(11.40 \pm 0.27$ y $7.84 \pm 0.77$, respectivamente) y $b^{*}(4.50 \pm 0.18$ y 11.51 \pm 0.12 , respectivamente). Por otro lado, se mencionan los valores para $L^{*}(31.26 \pm 0.46$ y $38.03 \pm 0.44$ respectivamente), $a^{*}(9.77 \pm 0.26$ y $7.78 \pm 0.98$, respectivamente) y $b^{*}(6.48 \pm 0.37$ y $10.71 \pm 0.58$ respectivamente) de las zanahorias con tratamiento térmico. Se observó que la luminosidad $\left(L^{*}\right)$ de la zanahoria roja no hubo diferencia significativa entre la muestra sin tratamiento y la muestra hervida, un comportamiento similar se presentó en la zanahoria naranja. Los valores de $a^{*}$ en ambas muestras disminuyeron con el tratamiento térmico en comparación con la muestra no tratada. En el parámetro $b^{*}$, los valores de la zanahoria roja aumentaron, mientras que en la zanahoria naranja hubo un descenso. Como se ha mencionado anteriormente, los compuestos responsables de la coloración de la zanahoria, son los carotenoides y de acuerdo al tratamiento aplicado, no hubo un cambio notable de color debido al corto tiempo en que la hortaliza fue sometida, ya que este tratamiento inactiva las enzimas, protegiendo así el color. ${ }^{40,4}$

\section{Compuestos antioxidantes}

Los antioxidantes son un grupo de sustancias que se pueden presentar de manera exógena y endógena con respecto al sustrato oxidable, los cuales retrasan o previenen significativamente la oxidación de este, como sustrato oxidable se le considera a casi todas las moléculas orgánicas e inorgánicas que se encuentran en las células vivas, como proteínas, lípidos, hidratos de carbono y las moléculas de ADN. ${ }^{41}$ Vegetales como la zanahoria contienen compuestos antioxidantes como los polifenoles, flavonoides, ácido ascórbico y carotenos. ${ }^{7}$

\section{Compuestos fenólicos}

Los compuestos fenólicos son una clase de compuestos orgánicos, que se encuentran en las plantas como metabolitos secundarios en diferentes concentraciones. ${ }^{42}$ En un estudio reportado por Wang et al. ${ }^{4}$ evaluaron el contenido de fenoles totales (CFT) en la zanahoria entera y los valores fueron de $1.75 \mathrm{mg}$ EAG (equivalentes de ácido gálico) $/ 100 \mathrm{~g}$ en ps (peso seco). Hussein et al. ${ }^{43}$ reportaron el contenido de fenoles totales de $87.4 \pm 2.37$ $\mathrm{mg}$ EAG/100 g ps y flavonoides totales de $35.9 \pm 0.97 \mathrm{mg}$ CAT (equivalentes de catequina) $/ 100 \mathrm{~g}$ ps en cáscara de zanahoria cruda. En otro estudio, Embaby et al. ${ }^{44}$ evaluaron el contenido fenoles totales en jugo de zanahoria y reportaron $19.76 \pm 0.527 \mathrm{mg}$ EAG/100 mL. Actualmente no existe una Norma sobre el consumo de compuestos fenólicos. Sin embargo, Corrêa et al. ${ }^{45}$ mencionan que el consumo de compuestos fenólicos es de $1 \mathrm{~g} /$ día. De acuerdo a lo anterior, $100 \mathrm{~mL}$ de jugo de zanahoria, estarían cubriendo el $1.97 \%$ del consumo diario de estos compuestos.

Por otro lado, a la zanahoria entera se le aplicó tratamiento de escaldado $\left(99^{\circ} \mathrm{C}\right)$ a diferentes tiempos $(2$, $4,6$ y $8 \mathrm{~min})$. Se observó que a mayor tiempo de exposición, los compuestos fenólicos disminuyeron, ya que la muestra escaldada por $8 \mathrm{~min}$ tuvo $11.71 \mathrm{mg}$ EAG/kg ps y el escaldado por 2 min presentó valores más altos (37.18 mg EAG/kg ps). ${ }^{4}$ La conservación de las propiedades antioxidantes de la zanahoria varía, ya que ocurre una degradación de compuestos bioactivos (como el ácido clorogénico, ácido cafeico, ácido ferúlico y ácido p-hidroxibenzoico) durante los procesos térmicos. ${ }^{12,4}$

Bilek et al. ${ }^{46}$ reportaron el contenido de fenoles totales y antocianinas en jugo de zanahoria pasteurizado (35.7 \pm $1.2 \mathrm{~g} \mathrm{EAG/kg} \mathrm{ms} \mathrm{(masa} \mathrm{seca))} \mathrm{y} \mathrm{se} \mathrm{comparó} \mathrm{con} \mathrm{el} \mathrm{jugo}$ sin tratamiento (43.3 $\pm 1.2 \mathrm{~g} \mathrm{EAG/kg} \mathrm{ms).} \mathrm{Se} \mathrm{observó} \mathrm{una}$ disminución significativa, al igual que en la cianidina 3xilosil (feruloilglucosil) galactósido y cianidina-3-xilosil (cumaroilglucosil) galactósido. La pérdida de compuestos fenólicos puede deberse a las interacciones con proteínas inducidas por el calor y a la estabilidad del catión flavilio de la antocianina a un $\mathrm{pH}$ de $1.5 .^{46}$

Suzme et al. ${ }^{47}$, reportaron una disminución en el contenido de flavonoides totales en el jugo de zanahoria negra (8277 $\pm 1735 \mathrm{mg}$ quercetina/100 g ps) después de la pasteurización (6945 $\pm 1546 \mathrm{mg}$ quercetina/100 g ps) (90- $95^{\circ} \mathrm{C}$ durante 90 segundos). Esto se debe a que los flavonoides son susceptibles a la degradación por los efectos térmicos. ${ }^{48}$ La temperatura rompe el enlace glucosídico en las moléculas combinadas fenol-azúcar de los flavonoides, debido a esto hay una disminución de estos compuestos bioactivos. ${ }^{49}$

La estimación sobre la absorción de compuestos antioxidantes en los alimentos, es a través de la metodología de bioaccesibilidad in vitro. ${ }^{16}$ Kamiloglu et al. $^{50}$ evaluaron la bioaccesibilidad de compuestos fenólicos en zanahoria negra y se observó un porcentaje de bioaccesibilidad del 4.9\%. El porcentaje tan bajo se debe a la inestabilidad de la estructura de los compuestos como el ácido clorogénico, cafeico, cafeoilquínico y neoclogénico. ${ }^{51,52}$

\section{Ácido ascórbico}

El ácido ascórbico (vitamina $\mathrm{C}$ ) es un compuesto orgánico soluble en agua y es la principal vitamina sintetizada por las plantas. ${ }^{53}$ Este compuesto es un antioxidante, ya que 
dona una sola reducción equivalente, y el radical que forma (monodehidroascorbato) reacciona con los radicales en lugar de los compuestos no radicales. ${ }^{54} \mathrm{La}$ vitamina $\mathrm{C}$ es comúnmente usada como un indicador para determinar la calidad nutricional de un producto, ya que es sensible a la degradación durante el procesamiento térmico. ${ }^{11}$ Existe evidencia que a partir de $30^{\circ} \mathrm{C}$ se va degradando la vitamina C. ${ }^{55}$ Por otra parte, Mendoza-Corvis et al. ${ }^{56}$ menciona que a $65^{\circ} \mathrm{C}$ la concentración de la vitamina $\mathrm{C}$ disminuye el $50 \%$ cuando está expuesta por 22.52 min al tratamiento térmico.

Cotruţ et al. ${ }^{53}$ cuantificaron el ácido ascórbico en frutas y vegetales frescos (manzana Golden y Jonatan, remolacha, lechuga, pimiento rojo, tomate cherry, naranja y zanahoria). El mayor contenido de ácido ascórbico lo obtuvo la naranja con 33.3-38.2 mg AA/100 g en pf (peso fresco), mientras que la zanahoria presentó un rango entre 1.90 y $9.92 \mathrm{mg} \mathrm{AA} / 100 \mathrm{~g}$ pf. Hussein et al. $^{43}$ analizaron el contenido de ácido ascórbico en la cáscara de zanahoria cruda y contenía $4.2 \pm 0.13 \mathrm{mg} \mathrm{AA} / 100$ g. Embaby et al. ${ }^{44}$ reportaron el contenido de ácido ascórbico en jugo de zanahoria y fue de $14.41 \pm 0.75 \mathrm{mg}$ $\mathrm{AA} / 100 \mathrm{~mL}$. Las diferencias entre las concentraciones de ácido ascórbico arriba mencionados, puede ser debido a los métodos de extracción, a la temporada en la que se sembró el vegetal y la elevada intensidad de luz a la que se expone el alimento durante ciertas estaciones del año, lo cual puede influir para incrementar el contenido de vitamina $C$ en los tejidos vegetales. ${ }^{57,58}$

Leong et al. ${ }^{55}$ realizaron un estudio en zanahoria (Nantes, Baby carrot y Egmont Gold) sobre la aplicación de un tratamiento térmico con temperaturas entre 30 y $80^{\circ} \mathrm{C}$ para evaluar su efecto en el contenido de vitamina C. A temperatura ambiente $\left(20^{\circ} \mathrm{C}\right)$, el primer cultivo reportó una concentración de vitamina $C$ de $330.70 \pm 31.16 \mu \mathrm{g} / \mathrm{g}$ ps, mientras que a la máxima temperatura $\left(80^{\circ} \mathrm{C}\right)$ se reportó $277.77 \pm 13.75 \mu \mathrm{g} / \mathrm{g}$ ps. El cultivo Baby carrot obtuvo $312.24 \pm 31.39 \mu \mathrm{g} / \mathrm{g}$ a temperatura ambiente y a $80^{\circ} \mathrm{C}$ fue un valor de $257.44 \pm 13.52 \mu \mathrm{g} / \mathrm{g}$ ps. Egmont Gold reportó $258.12 \pm 32.56 \mu \mathrm{g} / \mathrm{g}$ ps a $20^{\circ} \mathrm{C}$ y $318.82 \pm$ $18.40 \mu \mathrm{g} / \mathrm{g}$ ps a la temperatura más alta. Se pudo observar que en los dos primeros cultivos disminuyó la concentración del ácido ascórbico (vitamina C) cuando aumentó la temperatura, mientras que en el cultivo Egmond Gold se observó un comportamiento contrario, mientras aumentaba la temperatura, éste aumentaba su contenido de vitamina $\mathrm{C}$. Esto se debe a que la vitamina $\mathrm{C}$ es estable a temperaturas elevadas $\left(80^{\circ} \mathrm{C}\right)$, ya que a esa temperatura se inactiva la AAO (ácido-ascórbico oxidasa), la cual es responsable de la degradación del ácido ascórbico. ${ }^{55}$ Considerando la media de la Ingesta Diaria Recomendada (IDR) de vitamina C (82.5 mg/día) ${ }^{59}$, el consumo de $100 \mathrm{~g}$ de zanahoria tratada a $80^{\circ} \mathrm{C}$ estaría cubriendo el $38.64 \%$ de la IDR siendo buen aporte de esta vitamina, el cual podría contribuir con otros grupos de alimentos para una dieta correcta, ya que debe ser variada. $^{60}$

\section{Carotenoides}

Los carotenoides es un grupo de compuestos bioactivos que se encuentra en las zanahorias, siendo el $\alpha$-caroteno y $\beta$-caroteno los precursores metabólicos de la vitamina A. ${ }^{8}$ El carotenoide que mayormente se encuentra en las zanahorias es el $\beta$-caroteno, el cual es un tetraterpeno de 40 carbonos, simétrico y lineal, presenta dos anillos $\beta$ ionona, necesarios para realizar la síntesis de vitamina $A$ con ambos extremos (Figura 2). ${ }^{61}$
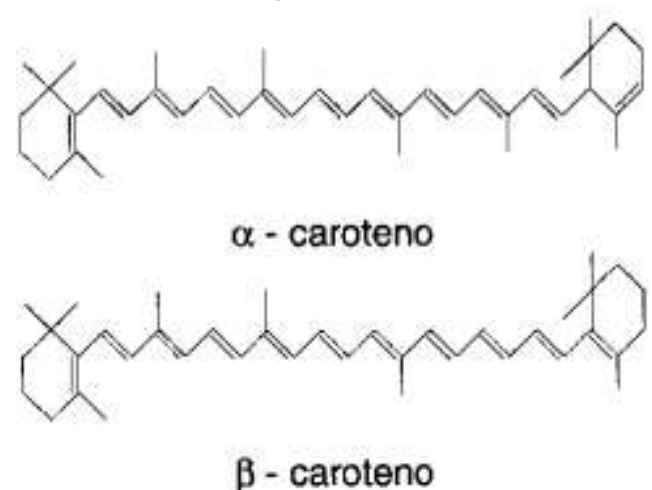

Figura 2. Estructura de $\alpha$-caroteno y $\beta$-caroteno ${ }^{61}$

Jeffery et al. ${ }^{2}$ analizaron el contenido de carotenoides en zanahoria entera, obteniendo valores de $2744.8 \pm 207.4$ y $4982.0 \pm 362.6 \mu \mathrm{g} / 100 \mathrm{~g}$ pf para $\alpha$-carotenos y $\beta$ carotenos, respectivamente. Por otro lado, Veda et al. ${ }^{37}$ realizaron un estudio en la zanahoria entera en donde aplicaron tratamientos térmicos como cocción a presión $\left(100^{\circ} \mathrm{C}, 15\right.$ p.s.i., $\left.10 \mathrm{~min}\right)(5134.5 \pm 75.3 \mu \mathrm{g} / 100 \mathrm{~g} \mathrm{pf})$, hervido por $10 \mathrm{~min}(6740.3 \pm 58.6 \mu \mathrm{g} / 100 \mathrm{~g} \mathrm{pf})$ y freído a sartén abierto durante $10 \mathrm{~min}$ a $100{ }^{\circ} \mathrm{C}(5926.4 \pm 106.6$ $\mu \mathrm{g} / 100 \mathrm{~g} \mathrm{pf)} \mathrm{y} \mathrm{el} \mathrm{porcentaje} \mathrm{de} \mathrm{carotenoides} \mathrm{disminuyó}$ (31.58, 10.18 y $21.03 \%$ ), respectivamente, en comparación con la zanahoria fresca (7504.0 \pm 80.4 $\mu \mathrm{g} / 100 \mathrm{~g} \mathrm{pf})$. Esto se debe a que los carotenoides son inestables al calor en presencia de la luz y oxígeno, los tratamientos térmicos provocan la isomerización de los carotenos convirtiendo el isómero de trans en la forma cis, disminuyendo así su actividad biológica. Por otro lado, al haber calentamiento se genera un reordenamiento molecular, causando la pérdida de valor nutricional. ${ }^{62}$

Lemmens et al. ${ }^{63}$, reportaron el contenido de $\beta$-carotenos en zanahorias frescas aplicando tres tratamientos. Los primeros dos fueron pretratados térmicamente mediante escaldado a baja temperatura $\left(60^{\circ} \mathrm{C}\right.$ por $\left.40 \mathrm{~min}\right)$ y alta temperatura $\left(90^{\circ} \mathrm{C}\right.$ por $\left.4 \mathrm{~min}\right)$, posteriormente ambos fueron cocinados a $95^{\circ} \mathrm{C}$ durante $20 \mathrm{~min}$. El tercer 
tratamiento consistió únicamente en el cocinado $\left(95^{\circ} \mathrm{C}-\right.$ $20 \mathrm{~min})$. Los resultados para las muestras pretratadascocinadas fueron de $87.70 \pm 2.75 \mu \mathrm{g} / \mathrm{g}$ pf y $111.01 \pm 5.58$ $\mu \mathrm{g} / \mathrm{g} \mathrm{pf}$, respectivamente, mientras que en la muestra cocinada se obtuvieron valores de $112.55 \pm 9.35 \mu \mathrm{g} / \mathrm{g}$ pf. El contenido de $\beta$-carotenos disminuyó notablemente con el escaldado a baja temperatura, en comparación con el escaldado a altas temperaturas y con la muestra que no se le aplicó pretratamiento térmico (cocinado). Lo anterior sugiere que el cocinar las zanahorias a elevada temperatura, puede mantener el contenido de $\beta$ carotenos, beneficiando su consumo a esas condiciones. Mayer-Miebach et al. ${ }^{64}$ realizaron un estudio en zanahoria donde se analizó el contenido de carotenoides. Se llevó a cabo el secado de rodajas de zanahorias a una temperatura entre 50 y $90^{\circ} \mathrm{C}$. La muestra fresca presentó $1107 \mathrm{mg} / \mathrm{kg} \mathrm{ms}$, mientras que las rodajas sometidas a temperaturas de $50,60,70$ y $90^{\circ} \mathrm{C}$ tuvieron valores de $997 \mathrm{mg} / \mathrm{kg} \mathrm{ms}, 1079 \mathrm{mg} / \mathrm{kg} \mathrm{ms}, 965 \mathrm{mg} / \mathrm{kg} \mathrm{ms}$ y 882 $\mathrm{mg} / \mathrm{kg} \mathrm{ms}$, respectivamente. Se observó que a esas temperaturas, el contenido de carotenoides disminuyó en comparación con la muestra fresca. En general, la aplicación de tratamientos térmicos afecta la estabilidad de los carotenoides, esto pudiera deberse a que estos compuestos son sensibles al calor, luz, oxígeno, oxidación, degradación y otros factores. ${ }^{65}$ Además de los tratamientos térmicos, la presencia de la fibra dietética también puede influir en la disminución del contenido de estos compuestos, ya que los carotenoides se encuentran dentro de la matriz fibrosa y complica la extracción. ${ }^{66}$

Por otro lado, se han realizado estudios de bioaccesibilidad in vitro de los carotenoides contenidos en la zanahoria cuando es sometida a tratamientos térmicos. Se ha reportado el porcentaje de bioaccesibilidad de $\alpha$ carotenos y $\beta$-carotenos $(20.2 \pm 3.4 \%$ y $21.6 \pm 3.9 \%$, respectivamente) en zanahoria completa. ${ }^{2}$ De acuerdo a la IDR del $\beta$-caroteno ( $4 \mathrm{mg} /$ día $)^{67}$, los carotenoides bioaccesibles $(1.076 \mathrm{mg}$ ) aportan el $26.9 \%$ del consumo diario recomendado al día. Sin embargo, se requiere revisar estudios sobre la biodisponibilidad de estos compuestos.

Lemmens et al. ${ }^{63}$, analizaron la bioaccesibilidad de carotenoides en zanahorias pretratadas empleado el escaldado a baja temperatura $\left(60^{\circ} \mathrm{C}\right.$ por $\left.40 \mathrm{~min}\right)$ y alta temperatura $\left(90^{\circ} \mathrm{C}\right.$ por $\left.4 \mathrm{~min}\right)$ y posteriormente fueron cocinadas a $95^{\circ} \mathrm{C}$ durante $20 \mathrm{~min}$, otras zanahorias sólo se cocinaron $\left(95^{\circ} \mathrm{C}\right.$ durante $\left.20 \mathrm{~min}\right)$. Con las condiciones de baja y alta temperatura de escaldado seguidos de cocción, se obtuvieron valores de $10.48 \pm 1.63 \mu \mathrm{g} / \mathrm{g}$ pf y $20.46 \pm 0.22 \mu \mathrm{g} / \mathrm{g}$ pf, respectivamente, mientras que la muestra cocinada presentó $22.37 \pm 2.17 \mu \mathrm{g} / \mathrm{g}$ pf. Se observó que disminuyó el porcentaje de bioaccesibilidad $(31.42 \pm 3.10 \%$ y $16.09 \pm 2.97 \%$, respectivamente) con respecto a la muestra cocinada (34.35 $\pm 4.74 \%)$. Lo anterior indica que los compuestos bioaccesibles son afectados cuando se someten a más de un tratamiento térmico a la vez. De acuerdo a los datos reportados de las muestras pretratadas, los $\beta$-carotenos estarían cubriendo el 26.2 y $51.15 \%$ de la IDR, respectivamente. La cocción a presión (1532.7 $\pm 38.4 \mu \mathrm{g} / 100 \mathrm{~g}$ peso fresco), el hervido (1716 $\pm 17.0 \mu \mathrm{g} / 100 \mathrm{~g}$ peso fresco) $\mathrm{y}$ el freído $(2280 \pm 92.9 \mu \mathrm{g} / 100 \mathrm{~g}$ peso fresco) en zanahoria entera provocó que el porcentaje de bioaccesibilidad de los carotenoides aumentara (29.80\%, 25.45\% y $38.47 \%$, respectivamente) en comparación con la zanahoria sin tratamiento (16.80\%). ${ }^{37}$ Los tratamientos térmicos mejoran la bioaccesibilidad de los carotenoides, debido a que la matriz del alimento se debilita y de este modo facilita su absorción. ${ }^{68}$ El freído presentó el mayor porcentaje de compuestos bioaccesibles, debido a la presencia de aceite vegetal como parte del tratamiento térmico, ya que el aceite es un triglicérido y al igual que el $\beta$-caroteno es de origen lipofílico. ${ }^{69}$

En un estudio realizado por Lemmens et al. ${ }^{70}$ se analizó la bioaccesibilidad in vitro de $\beta$-carotenos en pulpa de zanahoria. Se llevó a cabo bajo las condiciones de pasteurización de relevancia industrial $\left(90^{\circ} \mathrm{C}, 20 \mathrm{~min}\right)$, esterilización $\left(120^{\circ} \mathrm{C}, 20 \mathrm{~min}\right)$ y proceso de ultra alta temperatura (UHT) $\left(130^{\circ} \mathrm{C}, 50 \mathrm{~s}\right)$. Se observó que la que la pasteurización $(139.4 \mu \mathrm{g} / \mathrm{g} \mathrm{ms})$ y la UHT $(139.6 \mu \mathrm{g} / \mathrm{g}$ $\mathrm{ms})$ tuvieron un ligero aumento (1.08 y $1.23 \%$, respectivamente), mientras que la esterilización (152.2 $\mu \mathrm{g} / \mathrm{g} \mathrm{ms}$ ) presentó el mayor porcentaje de carotenoides bioaccesibles (10.36\%), en comparación con la muestra bioaccesible sin tratamiento térmico (137.9 $\mu \mathrm{g} / \mathrm{g} \mathrm{ms}$ ). Esto se debe a que el procesamiento térmico aumenta la micelarización del $\beta$-caroteno en la zanahoria. ${ }^{70}$

Zhang et al. ${ }^{71}$ evaluaron la bioaccesibilidad de carotenoides en zanahoria deshidratada. El secado se realizó a tres temperaturas $\left(60,70\right.$ y $\left.80^{\circ} \mathrm{C}\right)$ y se obtuvo $14.2 \pm 1.4 \%, \quad 15.9 \pm 2.1 \%$ y $10.7 \pm 0.5 \%$ de bioaccesibilidad, respectivamente. La deshidratación aumentó el \% de bioaccesibilidad respecto a la muestra cruda $(9.9 \pm 4.4 \%)$. Ribas et al. ${ }^{72}$ mencionan que puede ser por la elevada temperatura $\left(>60^{\circ} \mathrm{C}\right)^{71}$, ya que esto provoca modificaciones que pueden ser positivas para la biodisponibilidad de los compuestos antioxidantes, uno de ellos es la modificación de los polisacáridos de la membrana celular, esto sucede cuando las células se rompen durante el procesamiento. También se encuentran las proteínas, las cuales se modifican debido a la temperatura y $\mathrm{pH}$ afectando así su posible interacción. Por otro lado, los carotenoides se liberan fácilmente de la matriz del alimento con menor contenido de fibra dietética y tamaño de partícula, tal como se procesó la muestra en el estudio de la pulpa de zanahoria. ${ }^{70}$ Por lo tanto, los carotenoides al degradarse 
se transforman en compuestos más simples y puede conllevar a una mayor capacidad de extracción de compuestos durante la digestión. ${ }^{72}$

\section{Conclusiones}

Cuando la zanahoria es sometida a tratamientos térmicos como la pasteurización, cocción a presión, hervido, freído, escaldado, secado, esterilización y UHT, el contenido de compuestos fenólicos (fenoles y flavonoides) y carotenoides disminuye, mientras que el ácido ascórbico incrementa a temperaturas cercanas a $80^{\circ} \mathrm{C}$. Por otro lado, la bioaccesibilidad de los carotenoides aumenta en todos los tratamientos aplicados a excepción del escaldado. Sin embargo, la bioaccesibilidad de los compuestos depende del tipo de matriz alimentaria o a la interacción con otros compuestos que conforman el alimento.

\section{Referencias}

[1] Iorizzo M, Senalik A, Ellison S, Grzebelus D, Cavagnaro P, Allender C Brunet J, Spooner D, Van Deynce A, Simon P. Genetic structure and domestication of carrot (Daucus carota subsp. sativus) (Apiaceae). AJB 2013; 100(5): 930-938.

[2] Jeffery JL, Turner ND, King SR. Carotenoid bioaccessibility from nine raw carotenoid-storing fruits and vegetables using an in vitro model. J. Sci. Food Agric. 2012; 92(13): 2603-2610.

[3] Valero GT, Rodríguez AP, Ruiz ME, Ávila TJM, Varela MG. La alimentación española. Características nutricionales de los principales alimentos de nuestra dieta [En línea] 2da ed. Madrid, España: Ministerio de Agricultura, Pesca y Alimentación; 2018 [consulta: 24 abril 2021]. 658 p. Disponible

en:https://www.fen.org.es/storage/app/media/imgPublicaciones/2018/libro -la-alimentacion-espanola.pdf

[4] Wang H, Fang XM, Sutar PP, Meng JS, Wang J, Yu XL, Xiao HW. Effects of vacuum-steam pulsed blanching on drying kinetics, colour, phytochemical contents, antioxidant capacity of carrot and the mechanism of carrot quality changes revealed by texture, microstructure and ultrastructure. Food Chem. 2021; 338:127799.

[5] Servicio de Información Agroalimentaria y Pesquera. Zanahoria: sus mitos y realidades [En línea]. México: SIAP; 7 septiembre 2017 [consulta: 8 febrero 2020]. Disponible en: https://www.gob.mx/siap/es/articulos/zanahoria-sus-mitos-yrealidades?idiom $=\mathrm{es}$

[6] Kamiloglu S, Capanoglu E, Bilen FD, Gonzales GB, Grootaert C, Van de Wiele T, Van Camp J. Bioaccessibility of polyphenols from plantprocessing byproducts of black carrot (Daucus carota L.) J. Agric. Food Chem. 2015; 64(12): 2450-2458.

[7] Vargas VM, Figueroa BH, Tamayo CJ, Toledo LV, Moo Huchin V. Aprovechamiento de cáscaras de frutas: análisis nutricional y compuestos bioactivos. CIENCIA ergo-sum 2019; 26(2): 1-11.

[8] Gómez MA, Bandino E, Hormaza JI, Pilar CM. Characterization and the impact of in vitro simulated digestion on the stability and bioaccessibility of carotenoids and their esters in two Pouteria lucuma varieties. Food Chem. 2020; 316: 1-15
[9] Servicio de Información Agroalimentaria y Pesquera. Zanahoria: antioxidante a la vista [En línea]. México: SIAP; 6 junio 2018 [consulta: 8 $\begin{array}{lll}\text { febrero 2020]. Disponible } & \end{array}$ en: https://www.gob.mx/siap/articulos/zanahoria-antioxidante-a-lavista?idiom $=\mathrm{es}$

[10] López A. Industrialización de zanahoria. Rev. tecnoagro 2010; 52(1): $1-2$.

[11] Canacuan HGC, Murillo BLV, Santos LOEO. Efectos de los tratamientos térmicos en la concentración de vitamina $\mathrm{C}$ y color superficial en tres frutas tropicales. Rev. Lasallista Investig 2016; 13(1): 85-93.

[12] Cañas Z, Restrepo DA, Cortés M. Productos Vegetales como Fuente de Fibra Dietaria en la Industria de Alimentos. Rev Fac Nac Agron Medellín 2011; 64(1): 6023-6035.

[13] Valencia CS, Rodríguez HLF, Giraldo PGA. Cinética de la deshidratación y control de la oxidación en manzana Granny smith, mediante la aplicación de diferentes métodos de secado. Rev. Tumbaga 2011; 1(6):7-16

[14] Van Buggenhout S, Alminger M, Lemmens L, Colle I, Knockaert G, Moelants K, Hendrickx M. In vitro approaches to estimate the effect of food processing on carotenoid bioavailability need thorough understanding of process induced microstructural changes. Trends Food Sci. Technol. 2010; 21(12): 607-618

[15] Coronado HM, Vega LS, Gutiérrez TR, Vázquez FM, Radilla VC. Antioxidantes: perspectiva actual para la salud humana. Rev Chil Nutr 2015; 42(2): 206-212.

[16] Saura-Calixto F, Serrano J, Goñi I. Intake and bioaccessibility of total polyphenols in a whole diet. Food Chem. 2007; 101(2): 492-501.

[17] Organización de las Naciones Unidas para la Alimentación y la Agricultura. Producción orgánica de cultivos Andinos. Manual técnico [En línea]. Quito, Ecuador: Suquilanda VMB; 2011. [consulta: 3 noviembre 2020]. Disponible en: http://www.fao.org/fileadmin/user_upload/mountain_partnership/docs/1_p roduccion_organica_de_cultivos_andinos.pdf

[18] Leyva LF. Zanahoria roja y sus propiedades [En línea]. 2015. [consulta: 4 noviembre 2020]. Disponible en: https://todosobrezanahorias.blogspot.com/index.html.

[19] Leyva LF. Zanahoria morada [En línea]. 10 noviembre 2019. [consulta: 4 de noviembre 2020]. Disponible en: https://www.tuberculos.org/zanahoria/morada/

[20] Secretaría de Agricultura, Ganadería, Desarrollo Rural, Pesca y Alimentación. Zanahoria, una joya mundial. [En línea]. México: SAGARPA; 10 julio 2016 [consulta: 8 febrero 2020]. Disponible en: https://www.gob.mx/agricultura/es/articulos/zanahoria-una-joya-mundial.

[21] Del Rosamel C. Las zanahorias. Cultivo, cuidado y consejos prácticos. New York, USA: De Vecchi; 2019. 40 p.

[22] Agro Productos Agrícolas y Agroalimentarios. Zanahoria, taxonomía, y descripciones botánicas, morfológicas, fisiológicas y ciclo biológico [En línea]. España: 2013. [consulta: 4 marzo 2021]. Disponible en: https://www.agroes.es/cultivos-agricultura/cultivos-huertahorticultura/zanahoria/434-zanahoria-descripcion-morfologia-y-ciclo 
[23] Flaya M. Partes de la planta. Morfología de la planta zanahoria con hojas de color verde, tallo, raíz y títulos [En línea]. México: 23 septiembre 2018 [consulta: 24 abril 2021]. Disponible en: https://www.istockphoto.com/es/vector/partes-de-la-plantamorfolog\%C3\%ADa-de-la-planta-zanahoria-con-hojas-de-color-verdegm1036799486-277534992

[24] Dansa AM, Bougardt F, Nocera P. Perfil del mercado de zanahoria [En línea]. Argentina: Ministerio de agroindustria. Presidencia de la Nación; 2017 [consulta: 10 abril 2021]. Disponible en: https://www.magyp.gob.ar/sitio/areas/ss_mercados_agropecuarios/areas/h ortalizas/_archivos/000030_Informes/000996_Perfil\%20del\%20Mercado $\% 20 \mathrm{de} \% 20$ Zanahoria\%202017.pdf

[25] Zaccari VFI. Caracterización de seis cultivares de zanahorias (Daucus carota L.), crudas y cocidas al vapor, por color y contenido y bioaccesibilidad in vitro de $\beta$-carotenos y minerales [Tesis de Maestría en línea] Uruguay: Universidad de la República; 2010 [consulta: 10 septiembre 2020]. 117 p. Disponible https://www.colibri.udelar.edu.uy/jspui/bitstream/20.500.12008/1812/1/00 55zac.pdf

[26] Centro de Estudios para el Desarrollo Rural Sustentable y la Soberanía Alimentaria. Análisis de producción y consumo de hortalizas [En línea]. México: CEDRSSA; febrero 2020. [consulta: 28 abril 2021]. Disponible en: http://www.cedrssa.gob.mx/files/b/13/88Ana\%CC\%81lisis_produccio\%C C\%81n_consumo_hortalizas.pdf

[27] United States Department of Agriculture. National Nutrient Database for Standard Reference. Composition of Foods Raw, Processed, Prepared [En línea]. USA: USDA; 2008 [consulta: 20 marzo 2020]. Disponible en: http://www.nal.usda.gov/fnic/foodcomp/search/

[28] Escudero E, González P. La fibra dietética. Nutr Hosp. 2006; 21(2): $61-72$

[29] Tejada CF. Alteraciones del equilibrio del Potasio: Hipopotasemia Rev. Clin. Med. Fam. 2008; 2(3): 129-133

[30] Medina R, Moller C, Perdomo D, Bubis J. Aproximaciones de bioquímica clásica al estudio de la relación entre la estructura y la función de la rodopsina. Arch. Venez. Farmacol. Rer. 2008; 27(1): 5-13.

[31] Norma Oficial Mexicana NOM-242-SSA1-2009 [En línea]. Productos y servicios. Productos de la pesca frescos, refrigerados, congelados y procesados. Especificaciones sanitarias y métodos de prueba. Diario Oficial de la Federación. 25 agosto 2008 [consulta: 10 septiembre 2020]. Disponible

http://dof.gob.mx/nota_detalle_popup.php?codigo=5177531.

[32] Lee SW, Kim BK, Han JA. Physical and functional properties of carrots different cooked within the same hardness-range. LWT 2018; 93 346-353.

[33] Salas GF. Técnicas en cocina. Madrid, España: Síntesis; 2015. 252 p.

[34] Norma Oficial Mexicana NOM-184-SSA1-2002 [En línea]. Productos y servicios. Leche, fórmula láctea y producto lácteo combinado. Especificaciones sanitarias. Diario Oficial de la Federación. 16 junio 2000 [consulta: $\quad 10$ septiembre 2020]. Disponible en: http://www.salud.gob.mx/unidades/cdi/nom/184ssa12.html.

[35] Norma Oficial Mexicana NOM-130-SSA1-1995 [En línea]. Bienes y servicios. Alimentos envasados en recipientes de cierre hermético y sometidos a tratamiento térmico. Disposiciones y especificaciones sanitarias. Diario Oficial de la Federación. 23 febrero 1996 [consulta: 10 septiembre 2020]. Disponible en http://www.dof.gob.mx/nota_detalle.php?codigo $=4872315 \&$ fecha $=23 / 02 /$ 1996.

[36] Organización de las Naciones Unidas para la Alimentación y la Agricultura. Conservación de frutas y hortalizas mediante tecnologías y combinadas. Manual de capacitación [En línea]. Italia: Servicio de Tecnologías de Ingeniería Agrícola y Alimentaria (AGST); 2004. [consulta: 5 octubre 2020]. Disponible: http://www.fao.org/3/a-y5771s.pdf

[37] Veda S, Platel K, Srinivasan K. Enhanced bioaccessibility of $\beta$ carotene from yellow-orange vegetables and green leafy vegetables by domestic heat processing. Int. J. Food Sci. Technol. Int. 2010; 45(10): 2201-2207.

[38] Norma Oficial Mexicana NOM-187-SSA1/SCFI-2002 [En línea]. Productos y servicios. Masa, tortillas, tostadas y harinas preparadas para su elaboración y establecimientos donde se procesan. Especificaciones sanitarias. Información comercial. Métodos de prueba. Diario Oficial de la Federación. 18 agosto 2003 [consulta: 8 septiembre 2020]. Disponible en: http://www.salud.gob.mx/unidades/cdi/nom/187ssa1scfi02.html

[39] Mathias RK, Ah-Hen K. El color en los alimentos un criterio de calidad medible. Agro Sur 2014; 42(2): 39-48.

[40] Kaur A, Sogi DS. Effect of osmotic dehydration on physico-chemical properties and pigment content of carrot (Daucus carota $\mathrm{L}$ ) during preserve manufacture. J. Food Process. Preserv. 2016; 41(5): 1-6.

[41] Venereo J. Daño oxidativo, radicales libres y antioxidantes. Rev. Cub. Med. Mil. 2002; 31(2): 126-133.

[42] Tsimogiannis D, Oreopoulou V. Classification of Phenolic Compounds in Plants. En: Watson RR. Polyphenols in Plants: Isolation, Purification and Extract Preparation. 2da ed. London, United Kingdom: Academic Press; 2019; 263-284.

[43] Hussein AM, Kamil MM, Hegazy NA, Mahmoud KF, Ibrahim MA. Utilization of some fruits and vegetables by-products to produce high dietary fiber jam. Food Sci. Qual. Manag 2015; 37: 39-45.

[44] Embaby HES, Mokhtar SM. Impact of adding goldenberry (Physalis peruviana $\mathrm{L}$.) on some quality characteristics and bio-functional properties of pasteurized carrot (Daucus carota L.) nectar. Food Sci. Technol. 2019; 56(2): 966-975.

[45] Corrêa VG, Tureck C, Locateli G, Peralta RM, Koehnlein EA. Estimate of consumption of phenolic compounds by Brazilian population. Rev. Nut. Campinas. 2015; 28(2): 185-196

[46] Bilek SE, Yılma FM, Özkan G. The effects of industrial production on black carrot concentrate quality and encapsulation of anthocyanins in whey protein hydrogels. Food Bioprod. Process. 2017; 102: 72-80.

[47] Suzme S, Boyacioglu D, Toydemir G, Capanoglu E. Effect of industrial juice concentrate processing on phenolic profile and antioxidant capacity of black carrots. Int. J. Food Sci. Tech 2017; 49(3): 819-829.

[48] Sadilova E, Stintzing FC, Kammerer DR, Carle R. Matrix dependent impact of sugar and ascorbic acid addition on color and anthocyanin stability of black carrot, elderberry and strawberry single strength and from concentrate juices upon thermal treatment. Food Res. Int. 2009; 42(8): 1023 -1033 . 
[49] Gutiérrez TJ, Santiago SY, Hernández AD, Pinedo EJ, López BG López PC. Influencia de los métodos de cocción sobre la actividad antioxidante y compuestos bioactivos de tomate (Solanum lycopersicum L.). Nova Scientia. 2019; 11(22): 53-68.

[50] Kamiloglu S, Pasli AA, Ozcelik B, Van CJ, Capanoglu E. Influence of different processing and storage conditions on in vitro bioaccessibility of polyphenols in black carrot jams and marmalades. Food Chem. 2015; 186: 74-82.

[51] Parada J, Aguilera JM. Food Microstructure Affects the Bioavailability of Several Nutrients. J. Food Sci. 2007; 72(2): 21-32.

[52] Vallejo F, Gil A, Perez A, Garcia C. In vitro gastrointestinal digestion study of broccoli inflorescence phenolic compounds, glucosinolates, and vitamin C. J. Agric. Food Chem. 2004; 52: 135-138.

[53] Cotrut R, Bădulescu L. UPLC Rapid Quantification of Ascorbic Acid in Several Fruits and Vegetables Extracted Using Different Solvents. Agric. Agric. Sci. Procedia 2016; 10: 160-166.

[54] Njus D, Kelley PM, Tu YJ, Schlegel HB. Ascorbic Acid: The Chemistry Underlying Its Antioxidant Properties. Adv. Free Radical Biol. Med. 2020; 159: 37-43.

[55] Leong SY, Oey I. Effect of endogenous ascorbic acid oxidase activity and stability on vitamin C in carrots (Daucus carota subsp. sativus) during thermal treatment. Food Chem. 2012; 134(4): 2075-2085.

[56] Mendoza C, Fernando A, Hernández, Elvis J, Ruiz, LE. Efecto de Escaldado sobre el Color y Cinética de Degradación Térmica de la Vitamina $\mathrm{C}$ de la Pulpa de Mango de Hilacha (Mangífera indica var magdalena river). Inf. Tecnol. 2015; 26(3): 9-16.

[57] Dlamini CS. Provenance and family variation in seed mass and fruit composition in Sclerocarya birrea sub-species caffra. J. Hortic. For. 2011; 3(9): 286-293.

[58] Morris HJ, Weast CA, Lineweaver H. Seasonal variation in the enzyme content of eleven varieties of carrots. Bot. gaz 1996; 107(3): 362-372.

[59] National Institutes of Health. Datos sobre la Vitamina C [En línea] USA: NIH; 18 diciembre 2019. [consulta: 15 abril 2021. Disponible en: https://ods.od.nih.gov/pdf/factsheets/VitaminC-DatosEnEspanol.pdf

[60] Norma Oficial Mexicana NOM-043-SSA2-2005 [En línea]. Servicios básicos de salud. Promoción y educación para la salud en materia alimentaria. Criterios para brindar orientación. Diario Oficial de la Federación. 23 enero 2006 [consulta: 25 abril 2021]. Disponible en: http://www.salud.gob.mx/unidades/cdi/nom/compi/043ssa205.pdf

[61] Hauck SCA. Efecto del procesamiento térmico de zanahoria (D. carota) en la biodisponibilidad de $\beta$-caroteno obtenida mediante estudios in vitro. [Tesis de licenciatura]. Valparaíso, Chile: Universidad Técnica Federico Santa María; 2017, 84 p.

[62] Marx M. Effects of thermal processing on trans-cis-isomerization of $\beta$-carotene in carrot juices and carotene-containing preparations. Food Chem. 2003; 83(4): 609-617.

[63] Lemmens L, Van Buggenhout S, Oey I, Van Loey A, Hendrickx M. Towards a better understanding of the relationship between the $\beta$-carotene in vitro bio-accessibility and pectin structural changes: A case study on carrots. Food Res. Int. 2009; 42(9): 1323-1330.
[64] Mayer ME, Behsnilian D, Regier M, Schuchmann HP. Thermal processing of carrots: Lycopene stability and isomerisation with regard to antioxidant potential. Food Res. Int. 2005; 38(8-9): 1103-1108.

[65] Xiao YD, Huang WY, Li DJ, Song JF, Liu CQ, Wei QY, Yang QM. Thermal degradation kinetics of all-trans and cis-carotenoids in a lightinduced model system. Food Chem. 2018; 239: 360-368.

[66] Zhou J, Gugger E, Erdman J. The crystalline form of carotenes and the food matrix in carrot root decrease the relative bioavailability of beta-and alpha-carotene in the ferret model. J Am. Coll. Nutr. 1996; 15: 84-91.

[67] Institute of Medicine, Food and Nutrition Board. Beta-carotene and other carotenoids. Dietary reference intakes for vitamin C, vitamin E, selenium, and carotenoids. Washington, D.C.: National Academy Press; 2000: $325-400$.

[68] Van Het-Hof KH, De Boer BC, Tijburg LB. Carotenoid bioavailability in humans from tomatoes processed in different ways determined from the carotenoid response in the triglyceride-rich lipoprotein fraction of plasma after a single consumption and in the plasma after four days of consumption. J. Nutr. 2000; 130: 1189-1196.

[69] Yeum KJ, Russell RM. Carotenoid bioavailability and bioconversion. Annu. Rev. Nutr. 2002; 22: 483-504

[70] Lemmens L, Colle IJP, Van Buggenhout S, Van Loey AM, Hendrickx ME. Quantifying the Influence of Thermal Process Parameters on in Vitro $\beta$-Carotene Bioaccessibility: A Case Study on Carrots. J. Agric. Food Chem. 2011; 59(7): 3162-3167

[71] Zhang Z, Wei Q, Nie M, Jiang N, Liu C, Liu C, Xu L. Microstructure and bioaccessibility of different carotenoid species as affected by hot air drying: Study on carrot, sweet potato, yellow bell pepper and broccoli. LWT 2018; 96: 357-363

[72] Ribas-Agustí A, Martín-Belloso O, Soliva-Fortuny R, Elez-Martínez P. Food processing strategies to enhance phenolic compounds bioaccessibility and bioavailability in plant-based foods. Crit. Rev. Food Sci. Nutr. 2018; 58(15): 2531-2548. 